\title{
Phenotypic and Genotypic Characteristics of Biofilm Formation in Clinical Isolates of Acinetobacter baumannii
}

\author{
Zhaoyinqian Li* \\ Zixuan Ding* \\ Yao Liu \\ Xinrui Jin \\ Jingling Xie \\ Tingting $\mathrm{Li}$ \\ Zhangrui Zeng \\ Zhibin Wang \\ Jinbo Liu (D)
}

Department of Laboratory Medicine, the Affiliated Hospital of Southwest Medical University, Luzhou, 646000, Sichuan, People's Republic of China

*These authors contributed equally to this work
Correspondence: Jinbo Liu Department of Laboratory Medical, Affiliated Hospital of Southwest Medical University, 25 Taiping Street, Luzhou, 646000, Sichuan, People's Republic of China

$\mathrm{Tel} / \mathrm{Fax}+8608303165730$

Email Liulab2019@163.com
Background: Acinetobacter baumannii is an important pathogen in clinical infections, and biofilm formation is an effective way for $A$. baumannii to survive under external pressures. In this study, the aims were to examine the antimicrobial resistance, biofilm formation, and biofilm-specific resistance in clinical isolates of $A$. baumannii.

Materials and Methods: A total of 104 clinical A. baumannii isolates were collected from a large teaching hospital in Southwest China. The antibiotics susceptibilities were tested, and biofilm-forming ability was evaluated by crystal violet staining by confocal laser scanning microscopy (CLSM). Minimum inhibitory concentration (MIC), minimum bactericidal concentration (MBC), minimum biofilm inhibitory concentration (MBIC), and minimum biofilm eradication concentration (MBEC) of ciprofloxacin, meropenem, and ceftazidime were tested on selected strains by broth microdilution method. Biofilm-associated genes were detected by polymerase chain reaction (PCR), and expression of genes at planktonic stage and biofilm stage were analyzed by real-time reverse transcription PCR (RT-PCR).

Results: Multidrug-resistant (MDR) isolates accounted for $65.4 \%$, but no strain was resistant to tigecycline and polymyxin B. Moreover, non-MDR strains tended to form stronger biofilms than MDR strains, and a negative correlation between biofilm-forming ability and resistance profiles to each of tested antimicrobials were observed. The MBECs and MBICs of ciprofloxacin, ceftazidime, and meropenem were evidently increased compared with MICs and MBCs among all tested strains. Additionally, the biofilm formation ability of the csuDpositive strains was stronger than that of the $c s u D$-negative strains. The strains in MDR group had higher carrying rate of $\operatorname{csuA}$ and $\operatorname{csuD}$ genes than non-MDR group, while nonMDR strains possessed more ompA gene than MDR group. Finally, abaI gene was significantly up-regulated after biofilm formation.

Conclusion: These results revealed valuable data for the negative correlation between antimicrobial resistance and biofilm formation, as well as phenotypic and genotypic characteristics of biofilm formation in A. baumannii.

Keywords: Acinetobacter baumannii, biofilm formation, antimicrobial resistance, gene expression

\section{Introduction}

Acinetobacter baumannii is one of the most common nosocomial pathogen that causes several severe infections in critically ill patients, and a great number of multidrug-resistant (MDR) strains attract the attention of the whole world. ${ }^{1}$ The formation of biofilm contributes to $A$. baumannii adhere to various biological and non-biological surfaces, such as vascular catheters, cerebrospinal fluid shunts, and other medical devices. ${ }^{2}$ Biofilm 
is defining composed of extracellular polymeric substances (EPS) that surrounding microorganisms, which mediates microbial adhesion and makes a lifestyle that is totally different from the planktonic state. ${ }^{3}$ Indeed, biofilm formation presents a significantly higher antimicrobial resistance than planktonic cells, resulting in harder eradication and easier recurrence in biofilm-associated infections. ${ }^{4}$ Biofilm formation is a complex process and regulated by a variety of factors. Taking A. baumannii for example, biofilm associated protein (Bap) is involved in the initial adhering of biofilm, promoting the maturation of biofilm and maintaining the structure of mature biofilm. ${ }^{5,6}$ Moreover, synthesis and assembly of pili are encoded by $\operatorname{cs} U A / B A B C D E$ gene cluster to induce the initial adhesion process of biofilm. ${ }^{7}$ The BfmR-BfmS regulatory system senses the changes of external signals then affects the synthesis and secretion of pili, and induces the biofilm formation by regulating the $c s u A / B A B C D E$ gene cluster as well. ${ }^{8,9}$ The pgaABCD locus encodes the production of poly-beta-1-6$\mathrm{N}$-acetylglucosamine (PNAG), which is not only an important virulence factor but also essential for biofilm formation. ${ }^{10}$ The self-induced synthetase gene $a b a I$ is involved in the quorum sensing (QS) system and plays an important role in later stages of biofilm maturation. ${ }^{11,12}$ Outer membrane protein A (OmpA) is a well-known virulence factor that contributes to adhesion, invasion, and biofilm formation of A. baumannii. ${ }^{13}$ On the other hand, the latest research has reported that biofilm growth-associated repressor (BigR) directly or indirectly regulates the expression of genes related to epithelial cell adhesion and biofilm formation. ${ }^{14}$ Several biofilm-associated genes affect the antimicrobial susceptibility, indicating a relationship between biofilm formation and antimicrobial resistance. ${ }^{11}$

However, it is still unclear whether there is a quantitative correlation between biofilm formation and antimicrobial resistance. The aims of this study were to analyze the antimicrobial susceptibility, biofilm formation, biofilmspecific resistance, and biofilm-associated gene carrying rate of 104 clinical isolates of A. baumannii. Furthermore, the phenotype-genotype correlation between planktonic and biofilm stage was also evaluated.

\section{Materials and Methods}

\section{Identification and Antimicrobial} Susceptibility Testing (AST)

A total of 104 non-repetitive $A$. baumannii strains were isolated from January 2020 to April 2021 in the Affiliated Hospital of Southwest Medical University, and ATCC19606 was used as the reference strain in this study. Identification and antimicrobial resistance analysis were performed by the MicroScan Walk-Away 96 Plus system (Siemens, Germany), and results were interpreted according to the standards of the Clinical and Laboratory Standards Institute (CLSI) 2020-M100. ${ }^{15}$ Escherichia coli ATCC25922 and Pseudomonas aeruginosa ATCC27853 (purchased from China National Health Inspection Center) were used as quality control. Based on the obtained antimicrobial resistance profiles, the tested strains were classified into multidrug-resistant (MDR) group if 'the isolates are resistant to three or more antimicrobial classes', 16 and non-MDR group which the isolates are resistant to $0-2$ antimicrobial classes.

\section{Biofilm Formation Assay}

Biofilm formation ability was determined by crystal violet staining method as described previously, ${ }^{17}$ with slight modifications. Briefly, the overnight culture of each strain was diluted to final optical density of $600\left(\mathrm{OD}_{600}\right)=0.1$, and then $180 \mu \mathrm{L}$ of Luria-Bertani (LB) broth (Haibo, Qingdao, China) with 1\% glucose (Sigma, USA) and 20 $\mu \mathrm{L}$ of bacterial suspensions were inoculated into 96-well polystyrene microtiter plates (Costar \#3524, Corning, NY, USA) in triplicate. After $24 \mathrm{~h}$ incubation at $37{ }^{\circ} \mathrm{C}$, the plates were gently washed with phosphate-buffered saline (PBS) for three times and stained with $200 \mu \mathrm{L}$ of $0.1 \%$ crystal violet (Solarbio, Beijing, China) for $20 \mathrm{~min}$. After washing with PBS for three times again, the plates were solubilized with $200 \mu \mathrm{L}$ of $95 \%$ ethanol, followed by shaking slowly at room temperature for $20 \mathrm{~min}$. The absorbance was measured at $\mathrm{OD}_{570}$. The cut-off OD (ODc) was defined as three standard deviations (SD) above the mean OD of the negative control (LB broth only). Following were classification criteria: $\mathrm{OD} \leq \mathrm{OD}_{\mathrm{C}}$, non-biofilm producers $(-)$; $\mathrm{OD}_{\mathrm{C}}<\mathrm{OD} \leq 2 \times \mathrm{OD}_{\mathrm{C}}$, weak biofilm producers $(+) ; 2 \times \mathrm{OD}_{\mathrm{C}}<\mathrm{OD} \leq 4 \times \mathrm{OD}_{\mathrm{C}}$, moderate biofilm producers $(++)$; and $\mathrm{OD}>4 \times \mathrm{OD}_{\mathrm{C}}$, strong biofilm producers $(+++) .^{18}$

\section{Determination of Biofilm Using Confocal Laser Scanning Microscopy (CLSM)}

Four strains (two strong biofilm producers, one biofilmnegative producer, and ATCC19606) were selected for biofilm visualization by CLSM (Leica, Germany). In brief, strains were cultured in LB broth for $18 \mathrm{~h}$ and diluted to $0.5 \mathrm{McF}$ arland standard. One hundred microlitre suspension and $2 \mathrm{~mL}$ fresh LB broth were added in the 
petri dishes (FengRun, Beijing, China). After incubation at $37{ }^{\circ} \mathrm{C}$ for $24 \mathrm{~h}$, the petri dishes were washed three times with PBS and fixed with $2 \mathrm{~mL}$ of $2.5 \%$ glutaraldehyde for $1.5 \mathrm{~h}$. Then, the petri dishes were washed three times with PBS again and $200 \mu \mathrm{L}$ fluorescein isothiocyanate-concanavalin A (FITC-ConA) (Sigma, Germany) were added and stored at $4{ }^{\circ} \mathrm{C}$ for $30 \mathrm{~min}$. Two hundred microlitre propidium iodide (PI) (Sigma, Germany) were added and stored at $4{ }^{\circ} \mathrm{C}$ for $15 \mathrm{~min}$ after washing three times with PBS. Finally, the dishes were washed with PBS and sealed with $40 \%$ glycerol. FITC-conA binds to EPS of biofilm and reflects green fluorescence at the laser wavelength of $488 \mathrm{~nm} .{ }^{19}$ PI binds to bacterial DNA and emits red fluorescence at $543 \mathrm{~nm}^{20}$ If both lasers appear at the same time, an orange fluorescence will be shown. ${ }^{21}$

\section{Growth Curve Assay}

The growth of eight strong biofilm producers, eight weak biofilm producers, and ATCC19606 were evaluated. For each strain, $20 \mu \mathrm{L}$ of diluted overnight culture $(0.5$ McFarland standard) and $180 \mu \mathrm{L} \mathrm{LB}$ broth were added in 96-well plates and incubated at $37{ }^{\circ} \mathrm{C}$ for $24 \mathrm{~h}$, and the $\mathrm{OD}_{590}$ was detected by microplate reader every $2 \mathrm{~h}$ until $24 \mathrm{~h}$. After biofilm modeling on plates, the $\mathrm{OD}_{570}$ was measured by microplate reader every $4 \mathrm{~h}$ until the number of biofilm biomass decreased. All assays were repeated in triplicate.

\section{Minimum Inhibitory Concentration (MIC), Minimum Bactericidal Concentration (MBC), Minimum Biofilm Inhibitory Concentration (MBIC), and Minimum Biofilm Eradication Concentration (MBEC)}

Three antimicrobials that are commonly used for the treatment of A. baumannii infections were selected in the determination of MICs/MBCs and MBICs/MBECs. All of these nine strains were originally sensitive to tested antimicrobials. The MICs for ceftazidime, ciprofloxacin, and meropenem were assessed using broth microdilution method in 96-well plates according to the procedures described in CLSI 2020-M100 guideline. ${ }^{15}$ After MIC determination, $10 \mu \mathrm{L}$ aliquots from the wells with no observed bacterial growth were smeared on LB agar plates and incubated at $37^{\circ} \mathrm{C}$ for $24 \mathrm{~h}$, and the lowest concentration of antimicrobials that making no colony grows on plates was considered as MBC. AST at biofilm stage was performed according to the procedures described previously. ${ }^{22}$ Biofilm was established in 96-well plates for $24 \mathrm{~h}$, and planktonic cells were removed by sterile deionized water. Each well was treated with $200 \mu \mathrm{L}$ of LB broth containing serially diluted antimicrobials. The plates were incubated for $24 \mathrm{~h}$ at $37{ }^{\circ} \mathrm{C}$. The minimum antimicrobial concentration at which $\mathrm{OD}_{600}<0.1$ was determined as the MBIC.

Following the MBIC examination, the plates were washed with sterile deionized water to remove planktonic cells and antibiotics. Two hundred microlitre of LB broth was added to each well, and the plates were incubated at $37^{\circ} \mathrm{C}$ for $24 \mathrm{~h}$. The lowest concentration at which $\mathrm{OD}_{600}<$ 0.1 was considered as the MBEC. All experiments were performed in triplicate.

\section{Detection of Biofilm-Associated Genes by Polymerase Chain Reaction (PCR) and Real-Time Reverse Transcription PCR (RT-PCR)}

The DNA template was prepared by boiling bacteria. ${ }^{23}$ The biofilm-associated genes of all 104 clinical strains were detected by PCR, including bap, ompA, bigR, bfmS, bfmR, csuA, csuB, csuC, csuD, csuE, csuAB, pgaD, and abaI (Table S1). The procedures were followed by previous study with slight modifications. ${ }^{24}$ Part of nucleotide sequences of PCR products were analyzed by Beijing Qingke Biotechnology Co. Ltd. The results were compared with sequences that retrieved from GenBank database by the BLAST online tool at www.ncbi.nlm.nih.gov. Moreover, eight strong biofilm producers were selected to detect the expression level of 13 biofilm-associated genes by RT-PCR according to the protocol described previously. ${ }^{25}$ The fold change of mRNAs expression was calculated using the $2^{-\Delta \Delta \mathrm{Ct}}$ method, and the relative expression of each gene was normalized to the control sample (planktonic stage), which was assigned a value of 1 arbitrary unit. ${ }^{26}$ All assays were performed in triplicate.

\section{Statistical Analysis}

Statistical analyses and graphs were performed using GraphPad Prism 8.0.1 (GraphPad Software Inc, USA), Origin 2018 (OriginLab, USA) and SPSS 24.0 (SPSS Statistics, Inc., Chicago, IL, USA). Based on the distribution and homogeneity of the variances, the OD values were expressed as the mean \pm SD or median (interquartile 
range, IQR). Spearman's rank correlation test was used for intergroup comparison. Mann-Whitney $U$-test is used to analyze the carrying situation of 13 genes and biofilm formation capacity. Chi-square test was used to compare the positive rates of 13 genes and the number of genes carried in different resistance types of strains. Student's $t$-test was used for gene expression in planktonic stage and biofilm stage. In all analyses, a two-sided significance level of $<0.05$ was considered as statistically significant.

\section{Results}

\section{Distribution and Antimicrobial} Susceptibility Profiles of Clinical A. baumannii Strains

One hundred and four clinical A. baumannii isolates were collected from 18 departments within the major of intensive care unit $(27,26.2 \%)$ and department of neurology (22, 21.4\%). Furthermore, the majority of specimens were obtained from sputum, accounting for $72.8 \%$, while only $12.6 \%, 7.7 \%, 4.9 \%$, and $2 \%$ specimens were isolated from secretion, blood, cerebrospinal fluid, and urine, respectively. The AST results for 18 antimicrobials are shown in Figure 1. Most of isolates $(68,65.4 \%)$ were resistance to ciprofloxacin and piperacillin/tazobactam, followed by levofloxacin $(67,64.4 \%)$, cefepime $(67,64.4 \%)$, meropenem $(67,64.4 \%)$, imipenem $(67,64.4 \%)$, ceftazidime $(67$, $64.4 \%)$, ceftriaxone $(65,62.5 \%)$, cefotaxime $(65,62.5 \%)$, piperacillin (62, 59.6\%), ampicillin/sulbactam (61, $58.7 \%)$, ticarcillin/clavulanic acid $(60,57.7 \%)$, gentamicin $(54,51.9 \%)$, tobramycin $(52,50 \%)$, amikacin $(52,50 \%)$, and tetracycline $(30,28.8 \%)$. More than $65.4 \%$ of the isolates exhibited multidrug resistance (Figure 1B), but no strain was resistant to tigecycline and polymyxin $\mathrm{B}$.

\section{Biofilm Formation Ability and Growth Curve Analysis}

The biofilm-forming capacity of each isolate was summarized. The $\mathrm{OD}_{570}$ values for the reference strain (ATCC19606) and negative control were $0.84 \pm 0.12$ and $0.14 \pm 0.008$, respectively (Table $\mathrm{S} 2$ ). The $\mathrm{OD}_{570}$ values for the clinical isolates ranged from $0.14 \pm 0.01$ to $2.54 \pm$ 0.26 , and the IQR was $0.58(0.36,1.04)$. It is worth noting that $99 \%$ of these strains can form biofilm, of which $51.5 \%$ were strong biofilm producers. In addition, 35.6\% strains produced stronger biofilm than ATCC19606. No significant difference was observed between eight strong and eight weak biofilm producers in growth curves, indicating that the difference in biofilm formation was not due to growth rate (Figure 2). Furthermore, four strains were selected to observe the biofilm formation ability using CLSM. The strongest biofilm-forming strain 20047 reflected the highest fluorescence intensity, followed by strain 20192 and ATCC19606, and biofilm-forming negative strain 19014 presented the lowest fluorescence intensity (Figure 3A). The fluorescence intensities under CLSM were consistent with the $\mathrm{OD}_{570}$ values obtained by crystal violet staining (Figure 3B).

\section{Correlation Between Antimicrobial}

\section{Resistance and Biofilm Formation Ability}

Spearman's rank correlation analysis determined that nonMDR strains tended to form stronger biofilms than MDR strains $\left(r_{s}=-0.212, P=0.03\right.$; Table 1$)$, suggesting a negative correlation between biofilm formation capacity and antimicrobial resistance phenotypes. Moreover, the results revealed that in addition to tigecycline and polymyxin $\mathrm{B}$, susceptible strains could form stronger biofilm than resistant strains, indicating a negative correlation between biofilm-forming ability and resistance profiles to each of tested antimicrobials $\left(r_{s}=0.204-0.288, P=0.003-\right.$ 0.037; Table 2)

\section{Comparison of MICs/MBICs and MBCs/ MBECs}

The MICs and MBICs of three antimicrobials (ceftazidime, ciprofloxacin, and meropenem) were tested against nine strains with different biofilm-forming ability (three strong biofilm producers, three moderate biofilm producers, and three weak biofilm producers), and all strains were sensitive to these three antibiotics being investigated. MBICs for biofilms increased drastically compared with MIC for planktonic cells among all tested strains (Table 3). The increases ranged from 256- to 4096-fold for ciprofloxacin, 16- to 1024-fold for meropenem, and 128- to 1024-fold for ceftazidime. Indeed, when biofilms were established, the antimicrobial susceptibility profiles of all strains changed from sensitive to resistance.

Furthermore, MBCs and MBECs of these nine isolates were also evaluated. Undoubtedly, the MBECs were much higher than the MBCs among all tested strains (Table 4). Up to a 4096-fold increase in the concentration of ciprofloxacin and up to a 1024-fold increase in the concentration of ceftazidime and meropenem were required to eradicate biofilms compared with planktonic cells. 


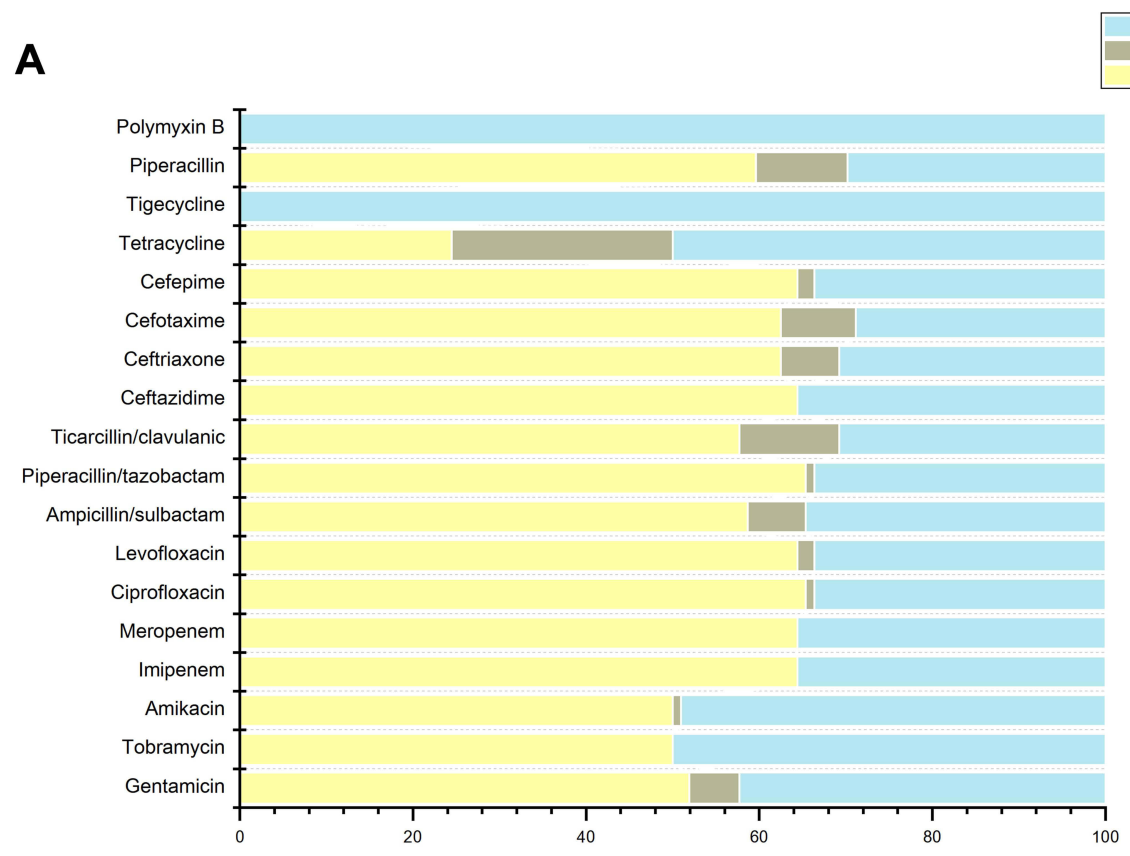

B

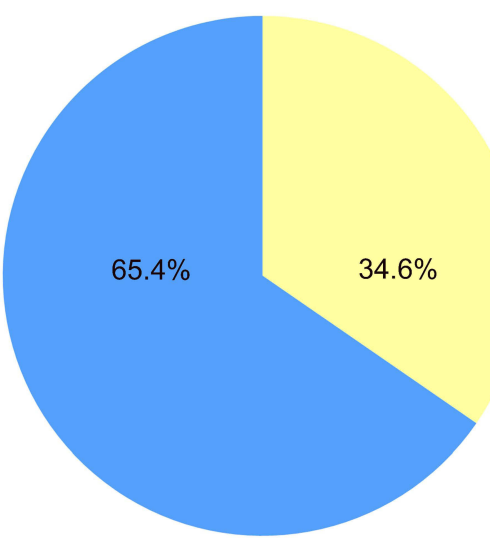

non-MDR

MDR

Total $=104$

Figure I Antimicrobial susceptibility profiles of clinical A. baumannii isolates. (A) Resistance rate of all strains to 18 antimicrobials; (B) approximately $65.4 \%$ of isolates exhibited MDR (including XDR).

Abbreviations: MDR, multiple drug resistance; XDR, extensively drug resistance.

\section{Distribution and Expression of Biofilm-} Associated Genes in Clinical A. baumannii

\section{Strains}

In order to further explore the correlation between biofilmforming ability and antimicrobial resistance, the presence of 13 reported biofilm-associated genes was detected by using PCR. The results showed that $b f m R(98,94.2 \%)$ was occurred mostly, followed by $\operatorname{csuC}(95,91.3 \%), \operatorname{csu} E(95,91.3 \%), \operatorname{big} R$ (92, 88.5\%), csuAB (91, 87.5\%), csuB (90, 86.5\%), OmpA (89,
$85.6 \%)$, bfmS (88, 84.6\%), bap (85, 81.7\%), pgaD (80, $76.9 \%), \operatorname{csuA}(76,73.1 \%), \operatorname{csuD}(73,70.2 \%)$, and $a b a I$ (73, $70.2 \%$ ). Twenty-five clinical isolates contained all 13 tested genes, including 23 strong biofilm producers and two moderate biofilm producers (Table S3). Remarkably, the biofilm formation ability of the $\operatorname{csuD} D$-positive strains was stronger than that of the $c s u D$-negative strains $(\mathrm{U}=365, P<0.01)$.

The harboring rates of 13 biofilm-associated genes among two groups were evaluated by Chi-square test. 
A

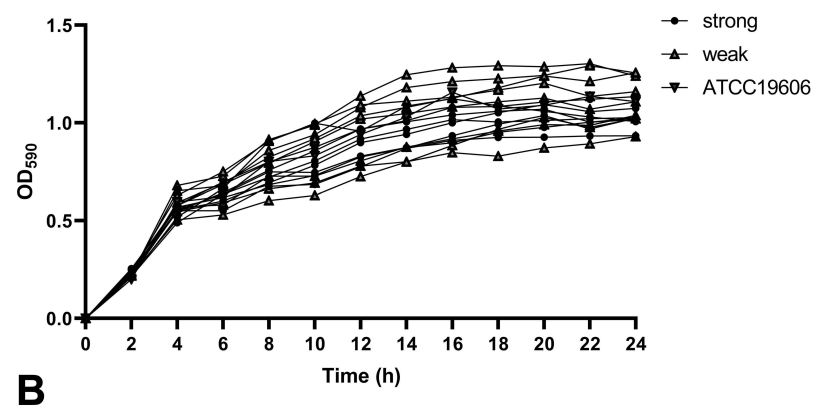

B

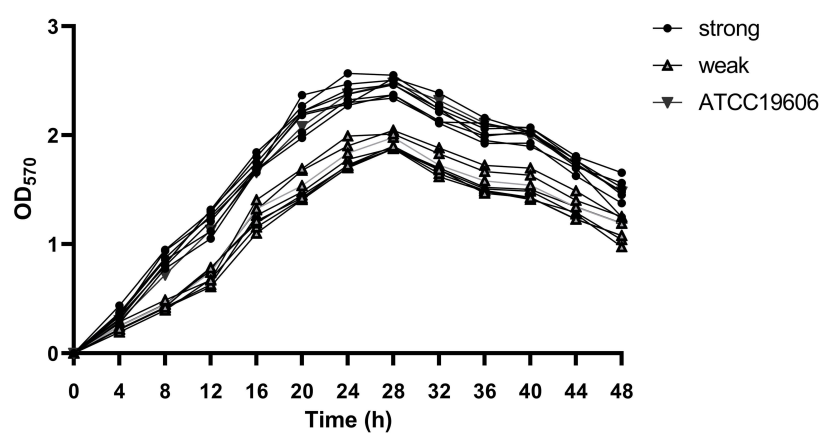

Figure 2 Growth curves and biofilm growth curves in representative A. baumannii strains. (A) Growth curves of 17 representative A. baumannii strains at planktonic stage, and $O D_{590}$ values of each strain were measured every $2 \mathrm{~h}$ until $24 \mathrm{~h}$; (B) growth curves for 17 representative $A$. baumannii strains at biofilm stage, and $O D_{570}$ values of each strain were measured every $4 \mathrm{~h}$ until $48 \mathrm{~h}$. The data were presented as the average of three measurements. Error bars (standard deviation $<10 \%$ ) were omitted for simplicity.
Three genes were significantly related to antimicrobial resistance. In detail, the strains in MDR group had higher carrying rate of $\operatorname{csuA}$ and $\operatorname{csuD}$ genes than non-MDR group $(P<0.05)$; however, the non-MDR strains possessed more ompA gene than MDR group $(P=0.002)$ (Figure 4A).

Eight strong biofilm-forming strains that carrying 13 biofilm-associated genes were selected to investigate the relationship between biofilm-associated genes and biofilm formation ability (Table S4). The RT-PCR results showed that the expression of abaI gene in the biofilm stage was significantly up-regulated compared with that in the planktonic stage $(P<0.001)$ (Figure 4B), while no significant difference was found for the rest of the 12 genes between planktonic stage and biofilm stage.

\section{Discussion}

Drug-resistant $A$. baumannii shows superior ability to disseminate worldwide, and the proportion of extreme drug resistance (XDR) A. baumannii has increased from less than $4 \%$ in 2000 to more than $60 \%$ recently, even close to $90 \%$ in some regional nosocomial settings. ${ }^{27}$ According to the National Institutes of Health and the Center for Disease and Prevention, it is estimated that $65 \%$ to $80 \%$

A

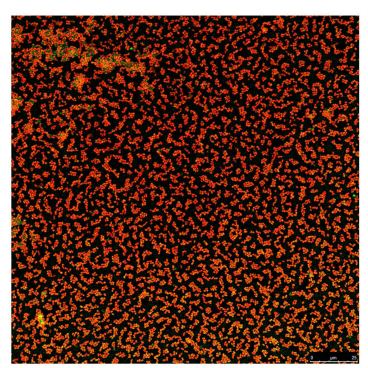

20047

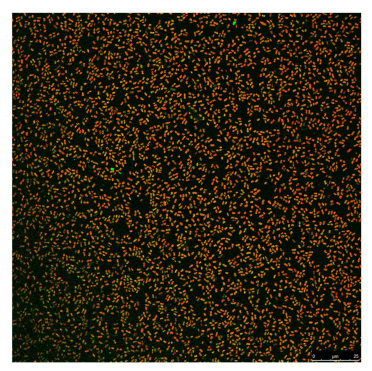

ATCC19606

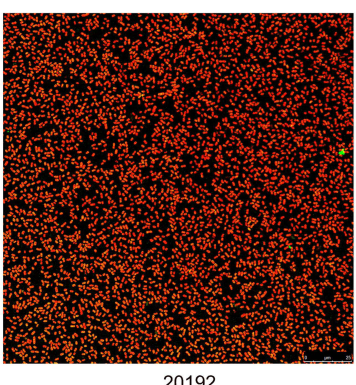

20192

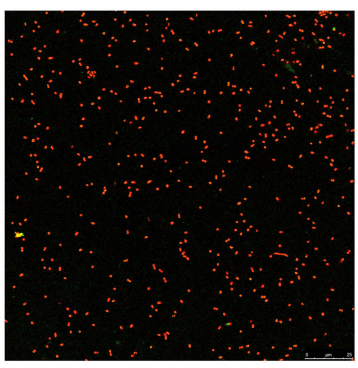

19014
B

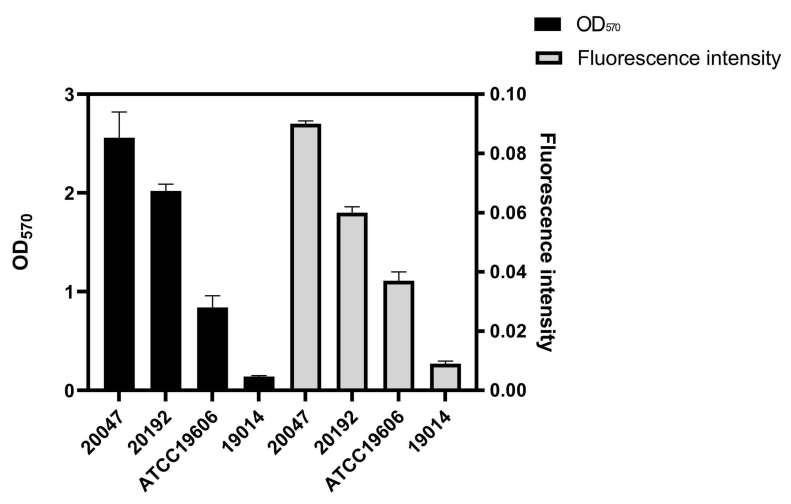

Figure 3 Evaluation of biofilm formation capacity of strains by confocal laser scanning microscopy. (A) Biofilm Images of four representative A. baumannii strains under CLSM (B) Comparison of biofilm formation capabilities between crystal violet and CLSM. Data were expressed as mean \pm SD; fluorescence intensity represents CLSM results and $O D_{570}$ indicates crystal violet results. 
Table I Biofilm Formation Ability of Strains with Different Antimicrobial Resistance

\begin{tabular}{|l|c|c|c|c|}
\hline Resistance Profile & $\mathbf{N}$ & OD $_{570}$ & $\boldsymbol{r}_{\mathbf{s}}$ & $\boldsymbol{P}$ \\
\hline Non-MDR & 36 & $0.98(0.36,1.47)$ & -0.212 & 0.03 \\
MDR & 68 & $0.55(0.36,0.85)$ & & \\
\hline
\end{tabular}

Notes: $\mathrm{OD}_{570}$ : The absorbance value at the wavelength of $570 \mathrm{~nm}$. Data was shown in median (interquartile range, IQR).

Table 2 Correlation Between Biofilm Formation Ability and Antimicrobial Resistance to 18 Antimicrobials

\begin{tabular}{|c|c|c|c|c|c|}
\hline \multirow[t]{2}{*}{ Antimicrobial Category } & \multirow[t]{2}{*}{ Antimicrobial Agent } & \multicolumn{2}{|c|}{$O D_{570}$} & \multirow[t]{2}{*}{$r_{s}$} & \multirow[t]{2}{*}{$P$} \\
\hline & & $\mathbf{S}$ & $\mathbf{R}$ & & \\
\hline \multirow[t]{3}{*}{ Aminoglycosides } & Gentamicin & $0.7 \mid(0.34, \mid .28)$ & $0.55(0.38,0.84)$ & -0.211 & 0.032 \\
\hline & Tobramycin & $0.82(0.36,1.33)$ & $0.53(0.37,0.82)$ & -0.211 & 0.032 \\
\hline & Amikacin & $0.84(0.36,1.34)$ & $0.53(0.37,0.82)$ & -0.214 & 0.03 \\
\hline \multirow[t]{2}{*}{ Carbapenemase } & Imipenem & $0.92(0.36, I .43)$ & $0.53(0.37,0.85)$ & -0.215 & 0.029 \\
\hline & Meropenem & $0.92(0.36,1.43)$ & $0.53(0.37,0.85)$ & -0.215 & 0.029 \\
\hline \multirow[t]{2}{*}{ Fluoroquinolones } & Ciprofloxacin & $0.92(0.36,1.34)$ & $0.55(0.37,0.87)$ & -0.213 & 0.031 \\
\hline & Levofloxacin & $0.92(0.36,1.34)$ & $0.55(0.37,0.87)$ & -0.213 & 0.031 \\
\hline \multirow[t]{3}{*}{ Penicillins $+\beta$-lactamase inhibitors } & Ampicillin/sulbactam & $0.98(0.36, I .47)$ & $0.69(0.49,0.72)$ & -0.239 & 0.018 \\
\hline & Piperacillin/tazobactam & $0.98(0.36,1.47)$ & $0.53(0.36,0.85)$ & -0.235 & 0.016 \\
\hline & Ticarcillin/clavulanic acid & $1.09(0.37,1.52)$ & $0.53(0.36,0.84)$ & -0.288 & 0.003 \\
\hline \multirow[t]{4}{*}{ Cephalosporins } & Ceftazidime & $0.92(0.36, I .43)$ & $0.53(0.37,0.85)$ & -0.215 & 0.029 \\
\hline & Ceftriaxone & $0.92(0.36,1.43)$ & $0.53(0.37,0.85)$ & -0.215 & 0.029 \\
\hline & Cefotaxime & $0.98(0.37,1.38)$ & $0.53(0.36,0.78)$ & -0.263 & 0.007 \\
\hline & Cefepime & $0.98(0.36,1.47)$ & $0.53(0.36,0.85)$ & -0.235 & 0.016 \\
\hline \multirow[t]{2}{*}{ Tetracyclines } & Tetracycline & $0.91(0.38, \mid .25)$ & $0.59(0.36,1.00)$ & -0.209 & 0.034 \\
\hline & Tigecycline & $0.57(0.36, \mathrm{I} . \mathrm{I})$ & 0 & NA & NA \\
\hline Penicillins & Piperacillin & $0.88(0.36, I, 30)$ & $0.53(0.37,0.78)$ & -0.204 & 0.037 \\
\hline Lipopeptides & Polymyxin B & $0.57(0.36,1.1)$ & 0 & NA & NA \\
\hline
\end{tabular}

Notes: $S$, sensitive; R, resistance; bold indicates $P<0.05$.

Table 3 Comparison of MICs and MBICs of A. baumannii Strains

\begin{tabular}{|c|c|c|c|c|c|c|c|c|c|}
\hline \multirow{2}{*}{ Strain } & \multicolumn{3}{|c|}{ Ciprofloxacin } & \multicolumn{3}{|c|}{ Meropenem } & \multicolumn{3}{|c|}{ Ceftazidime } \\
\hline & $\begin{array}{c}\text { MIC for } \\
\text { Planktonic } \\
\text { Cells } \\
(\mu \mathrm{g} / \mathrm{mL})\end{array}$ & $\begin{array}{c}\text { MBIC for } \\
\text { Biofilm } \\
\text { Cells } \\
(\mu \mathrm{g} / \mathrm{mL})\end{array}$ & $\begin{array}{c}\text { Fold } \\
\text { Change }\end{array}$ & $\begin{array}{c}\text { MIC for } \\
\text { Planktonic } \\
\text { Cells } \\
(\mu \mathrm{g} / \mathrm{mL})\end{array}$ & $\begin{array}{c}\text { MBIC for } \\
\text { Biofilm } \\
\text { Cells } \\
(\mu \mathrm{g} / \mathrm{mL})\end{array}$ & $\begin{array}{c}\text { Fold } \\
\text { Change }\end{array}$ & $\begin{array}{c}\text { MIC for } \\
\text { Planktonic } \\
\text { Cells } \\
(\mu \mathrm{g} / \mathrm{mL})\end{array}$ & $\begin{array}{c}\text { MBIC for } \\
\text { Biofilm } \\
\text { Cells } \\
(\mu \mathrm{g} / \mathrm{mL})\end{array}$ & $\begin{array}{c}\text { Fold } \\
\text { Change }\end{array}$ \\
\hline $20053^{a}$ & 0.125 & 32 & 256 & 0.5 & 8 & 16 & 8 & 1024 & 128 \\
\hline $2006 I^{a}$ & 0.0625 & 32 & 512 & 0.25 & 16 & 64 & 4 & 1024 & 256 \\
\hline $20068^{a}$ & 0.0625 & 32 & 512 & 0.5 & 16 & 32 & 2 & 1024 & 512 \\
\hline $20098^{b}$ & 0.0625 & 64 & 1024 & 0.25 & 16 & 64 & 4 & 1024 & 256 \\
\hline $20101^{\mathrm{b}}$ & 0.125 & 32 & 256 & 0.25 & 16 & 64 & 2 & 1024 & 512 \\
\hline $20110^{\mathrm{b}}$ & 0.125 & 32 & 256 & I & 16 & 16 & 4 & 1024 & 256 \\
\hline $20047^{c}$ & 0.125 & 512 & 4096 & 0.5 & 512 & 1024 & 4 & 4096 & 1024 \\
\hline $20180^{c}$ & 0.125 & 64 & 512 & 0.5 & 16 & 32 & 8 & 2048 & 256 \\
\hline $20250^{c}$ & 0.0625 & 64 & 1024 & I & 16 & 16 & 8 & 2048 & 256 \\
\hline
\end{tabular}

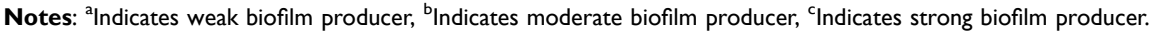


Table 4 Comparison of MBCs and MBECs of A. baumannii Strains

\begin{tabular}{|c|c|c|c|c|c|c|c|c|c|}
\hline \multirow[t]{2}{*}{ Strain } & \multicolumn{3}{|c|}{ Ciprofloxacin } & \multicolumn{3}{|c|}{ Meropenem } & \multicolumn{3}{|c|}{ Ceftazidime } \\
\hline & $\begin{array}{c}\text { MBC for } \\
\text { Planktonic } \\
\text { Cells } \\
(\mu \mathrm{g} / \mathrm{mL})\end{array}$ & $\begin{array}{c}\text { MBEC for } \\
\text { Biofilm } \\
\text { Cells } \\
(\mu \mathrm{g} / \mathrm{mL})\end{array}$ & $\begin{array}{c}\text { Fold } \\
\text { Change }\end{array}$ & $\begin{array}{c}\text { MBC for } \\
\text { Planktonic } \\
\text { Cells } \\
(\mu \mathrm{g} / \mathrm{mL})\end{array}$ & $\begin{array}{c}\text { MBEC for } \\
\text { Biofilm } \\
\text { Cells } \\
(\mu \mathrm{g} / \mathrm{mL})\end{array}$ & $\begin{array}{c}\text { Fold } \\
\text { Change }\end{array}$ & $\begin{array}{c}\text { MBC for } \\
\text { Planktonic } \\
\text { Cells } \\
(\mu \mathrm{g} / \mathrm{mL})\end{array}$ & $\begin{array}{c}\text { MBEC for } \\
\text { Biofilm } \\
\text { Cells } \\
(\mu \mathrm{g} / \mathrm{mL})\end{array}$ & $\begin{array}{l}\text { Fold } \\
\text { Change }\end{array}$ \\
\hline $20053^{a}$ & 0.25 & 128 & 512 & I & 128 & 128 & 16 & 2048 & 128 \\
\hline $20061^{a}$ & 0.125 & 64 & 512 & 0.5 & 32 & 64 & 8 & 2048 & 256 \\
\hline $20068^{a}$ & 0.125 & 64 & 512 & I & 32 & 32 & 4 & 2048 & 512 \\
\hline $20098^{\mathrm{b}}$ & 0.125 & 128 & 1024 & 0.5 & 32 & 64 & 8 & 2048 & 256 \\
\hline $20101^{b}$ & 0.25 & 64 & 256 & 0.5 & 128 & 256 & 4 & 2048 & 512 \\
\hline $20110^{b}$ & 0.25 & 64 & 256 & 2 & 256 & 128 & 8 & 2048 & 256 \\
\hline $20047^{c}$ & 0.25 & 1024 & 4096 & I & 1024 & 1024 & 8 & 8192 & 1024 \\
\hline $20180^{c}$ & 0.25 & 128 & 512 & I & 256 & 256 & 16 & 4096 & 256 \\
\hline $20250^{c}$ & 0.125 & 128 & 1024 & 2 & 64 & 32 & 16 & 4096 & 256 \\
\hline
\end{tabular}

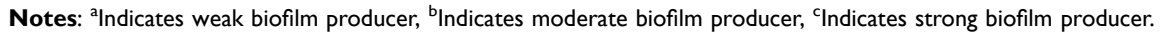

of the human infectious are caused by biofilm-forming bacteria. $^{28}$ The lifestyle changes from planktonic phenotype to biofilm is a highly regulated developmental process that depends on species-dependent environmental and genetic factors. ${ }^{4}$

This study evaluated the biofilm-forming ability of 104 clinical A. baumannii strains, and explored the effect of biofilm production on antimicrobial susceptibility. The MDR strains have reached $65.4 \%$, which increased the difficulty of infection treatment. The AST results revealed that all tested strains remained sensitive to polymyxin B and tigecycline, and it seemed that these two antimicrobials were the most effective strategy for MDR- $A$. baumannii infections. However, two meta-analyses discourage using tigecycline for the treatment of MDR-A. baumannii infections due to the higher in-hospital mortality and longer hospitalization when compared with other active antimicrobials. $^{29,30}$ Moreover, polymyxin B rarely used in clinical treatment because of the lack of pharmacokinetic/pharmacodynamic studies and highly renal toxicity. ${ }^{31}$

A

B

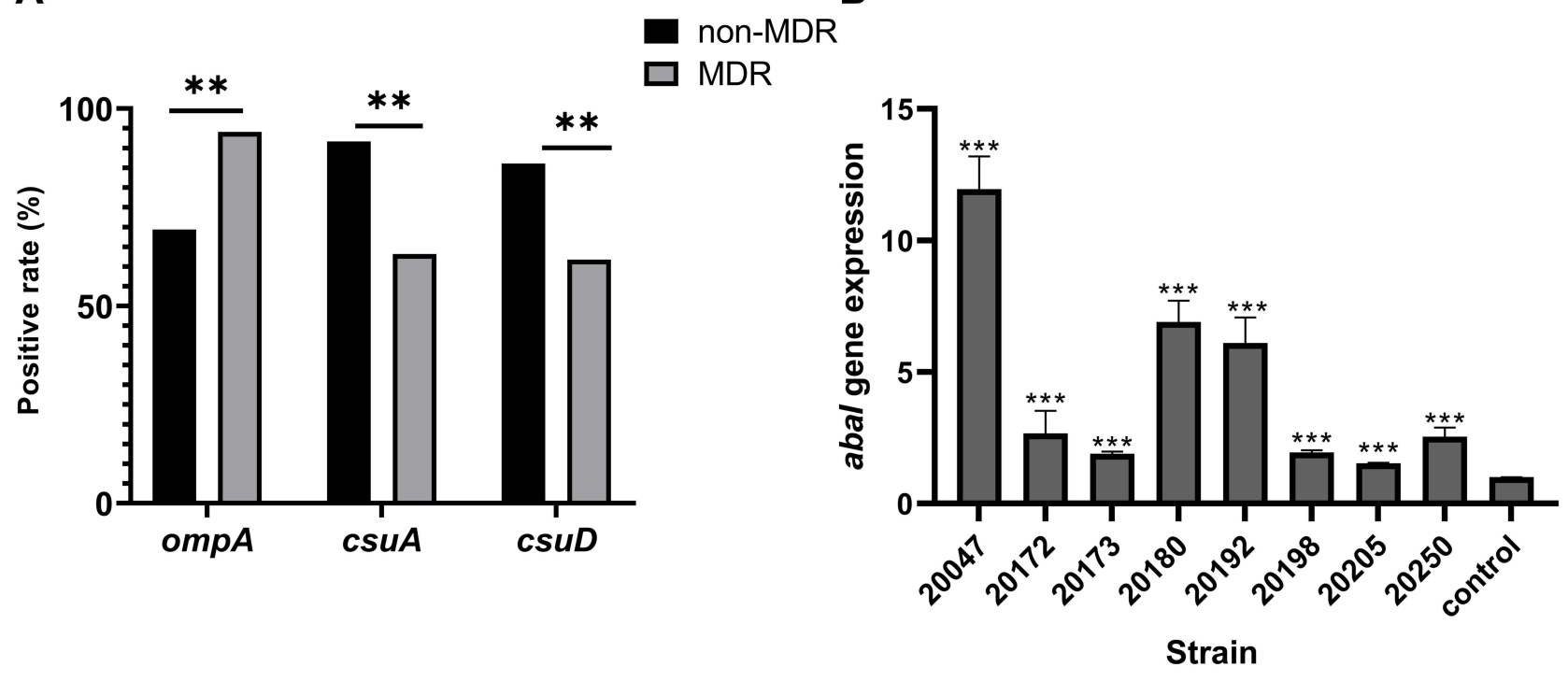

Figure 4 Role of biofilm-associated genes in antimicrobial resistance and biofilm of clinical A. Baumannii. (A) Percentage of biofilm-associated gene in different antimicrobialresistant strains; (B) the relative expression of $a b a l$ in eight selected A. Baumannii strains. Data were shown as fold changes between planktonic and biofilm stage, and control represented planktonic cells. Notes: $* * P<0.01, * * * P<0.001$ 
Therefore, the optimal treatment for MDR- $A$. baumannii infections have not been established so far. ${ }^{32}$

Then, the crystal violet staining results demonstrated that $99 \%$ strains have biofilm-forming ability and nearly one-half of strains can produce strong biofilms, which was consistent with the previous reports. ${ }^{24,33} \mathrm{Up}$ to date, there is still no regular pattern in the relationship between biofilm formation ability and bacterial antimicrobial resistance, and the conclusions between different researches were even contradictory. ${ }^{24,33-36}$ In our research, the nonMDR strains could produce stronger biofilm compared with MDR strains, indicating that the antimicrobial resistance and biofilm formation ability were negatively correlated in $A$. baumannii. Rodriguez-Bano et al have illustrated that biofilm-forming $A$. baumannii isolates were more sensitive to ciprofloxacin and imipenem, ${ }^{37}$ suggesting that these strains were not as dependent on antimicrobial resistance as non-biofilm-forming isolates for survival, which was consistent with our result. Moreover, a research has found that the biofilm-forming ability of ciprofloxacin-sensitive Pseudomonas aeruginosa was significantly promoted after exposure to ciprofloxacin, ${ }^{38}$ highlighting the importance of biofilms for the survival of sensitive bacteria. Therefore, it seems that the biofilm formation is another survival mechanism besides antimicrobial resistance. Furthermore, MIC/MBIC and $\mathrm{MBC} / \mathrm{MBEC}$ values confirmed that the resistance of isolates was greatly enhanced after biofilm formation, regardless of whether the isolates were originally sensitive or resistant to this antimicrobial. Remarkably, antimicrobials inhibit biofilms mainly through a complex penetration process, ${ }^{39}$ however, this still cannot complete remove the bacteria in the biofilm stage due to multifactorial tolerance of biofilms. ${ }^{40}$ For example, beta-lactam antimicrobials can be inactivated by beta-lactamases in the biofilm matrix, which restricts their penetration. Biofilm-stage bacteria with low metabolic activity can resistant to kinds of antimicrobials that target processes that occur in growing bacteria, such as cell wall synthesis and DNA replication. ${ }^{41}$ In addition, the expression of specific genes and dormant state persister cells are also responsible for the failure inhibition of biofilms. ${ }^{40,42}$

In addition to the correlation between antimicrobial resistance and biofilm formation, the relationship between biofilm formation and biofilm-associated genes expression was also evaluated in this study. All the biofilm-associated genes were detected in more than $70 \%$ of tested strains, bring with the most frequent gene $\operatorname{bfm} R(98,94.2 \%)$ as well as the least genes $c s u D$ and abaI (70.2\%). It has been known that the chaperone usher pili assembly system is essential for the initial steps of biofilm formation. ${ }^{43}$ Tomaras et al have certified that the inactivation of the $c s u E$ gene resulted in the disappearance of both pili formation and biofilm formation in A. baumannii. ${ }^{44}$ The gene $\operatorname{csuD}$ is part of the CsuA/BABCDE chaperone-usher pili assembly system, in this study, csuD-positive strains can form stronger biofilm than csuD-negative strains. This result was consistent with the previous study that indicating $c s u D$ was highly expressed in stronger biofilm producers. $^{45}$ Moreover, in this work, three genes had a obviously distributed regularity among strains with different antimicrobial-resistant profiles. With the increase of resistance, there was a growing positive rate of ompA and a decreasing positive rate of $\operatorname{csuA}$ and $\operatorname{csuD}$. Although CsuA/BABCDE system is able to enhance biofilm formation, the relationship between this system and antimicrobial resistance still not clear. OmpA is a major component of outer membrane proteins (OMPs) in $A$. baumannii, which is related to antimicrobial resistance, aggressiveness, and biofilm formation. ${ }^{13}$ Smani et al first determined that the deletion of ompA decreased the MICs of chloramphenicol, nalidixic and aztreonam by 8-, 2.67-, and 8-fold, respectively. ${ }^{46}$ Sanchez-Encinales et al demonstrated that overproduction of OmpA was an independent risk factor for nosocomial pneumonia, bacteremia, and higher mortality caused by $A$. baumannii infections. ${ }^{47} \mathrm{~A}$ previous study confirmed that omp $A$ measured by RT-PCR can be used as a rapid diagnostic factor for antimicrobialresistant $A$. baumannii due to the high consistency with MICs. $^{48}$ In A. baumannii, QS system is consists of AbaI/ AbaR system, which is closely interconnected with biofilm development. $^{12}$ The abaI gene encodes the self-induced synthase which activates the synthesis of AHL signals, and abaI mutant leads a greatly reduction of biofilm formation. $^{12,34}$ In this study, abaI was the only gene that up-regulated in biofilm stage; however, other tested genes did not show regular changes between planktonic and biofilm stages. A recent study has shown that the absence of AHL signal in shaking cultures (planktonic) seemed to be the result of low expression of abaI, while the AHL signal was significantly up-regulated under static conditions (biofilm). ${ }^{49}$ Niu et al have demonstrated that the biofilm formation ability of wild type and abaI mutant was no difference at $8 \mathrm{~h}$, but the biofilm formation ability of the mutant was significantly lower than that of wild type at $16 \mathrm{~h}$ and $24 \mathrm{~h}$, which was $40 \%$ and $41 \%$, respectively. 
These results suggest that the abaI-directed QS pathway is required for the later stages of biofilm maturation. ${ }^{12}$ The biofilms were established for $24 \mathrm{~h}$ in this study, and it might be the reason why only abaI was overexpressed and the expressions of other genes that contributed to the initial steps of biofilm formation were not significantly changed. ${ }^{1}$ Indeed, the mechanism of biofilm-associated genes in biofilm formation and antimicrobial resistance needed to be further explored.

\section{Conclusion}

In this study, most of $A$. baumannii have biofilm formation ability and high carrying rate of biofilm-associated genes. There was a negative correlation between biofilm formation ability and antimicrobial resistance, which was stronger biofilm formation occurring in non-MDR strains. Moreover, once the biofilm was formed, whether the biofilm was strong or weak, the antimicrobial resistance can be greatly improved. In addition, presence of $c s u D$ was significantly associated with biofilm-forming capacity. The $\operatorname{csu} A$ and $\operatorname{csuD}$ genes more occured in non-MDR strains, but $\operatorname{omp} A$ was more common in MDR strains. Notably, the expression of QS-related gene $a b a I$ up-regulated in biofilm stage. In fact, the number and type of biofilm-associated genes carried by biofilm-forming strains are quite different among different regions, ${ }^{11,50,51}$ indicating that the biofilm-forming ability is not only determined by the presence of biofilm-associated genes but a complex process depended on multiple factors. Deeper exploration of mechanisms of biofilm formation and biofilm-specific resistance in A. baumannii are highly needed.

\section{Ethics Approval and Consent to Participate}

This study was approved by the Institutional Review Board of affiliated hospital of southwest medical university (KY2020043). Written informed consent was obtained from all participants.

\section{Acknowledgments}

This work was supported by the grants from Sichuan Science and Technology Program (2021YFH001, 21ZDYF2148, 2019YFS0038), Southwest Medical University Science Program (2020ZRQNB025), and Luxian Government and Southwest Medical University Cooperation Program (2020LXXNYKD-04).

\section{Author Contributions}

All authors made substantial contributions to conception and design, acquisition of data, or analysis and interpretation of data; took part in drafting the article or revising it critically for important intellectual content; agreed to submit to the current journal; gave final approval of the version to be published; and agree to be accountable for all aspects of the work.

\section{Disclosure}

The authors declare that they have no conflicts of interest in this work.

\section{References}

1. Harding CM, Hennon SW, Feldman MF. Uncovering the mechanisms of Acinetobacter baumannii virulence. Nat Rev Microbiol. 2018;16 (2):91-102. doi: $10.1038 /$ nrmicro.2017.148

2. Greene C, Wu J, Rickard AH, Xi C. Evaluation of the ability of Acinetobacter baumannii to form biofilms on six different biomedical relevant surfaces. Lett Appl Microbiol. 2016;63(4):233-239. doi:10.1111/lam.12627

3. Flemming HC, Wingender J. The biofilm matrix. Nat Rev Microbiol. 2010;8(9):623-633. doi:10.1038/nrmicro2415

4. Hall CW, Mah TF. Molecular mechanisms of biofilm-based antibiotic resistance and tolerance in pathogenic bacteria. FEMS Microbiol Rev. 2017;41(3):276-301.

5. Amin M, Navidifar T, Shooshtari FS, et al. Association between biofilm formation, structure, and the expression levels of genes related to biofilm formation and biofilm-specific resistance of Acinetobacter baumannii strains isolated from burn infection in Ahvaz, Iran. Infect Drug Resist. 2019;12:3867-3881. doi:10.2147/ IDR.S228981

6. Brossard KA, Campagnari AA. The Acinetobacter baumannii biofilm-associated protein plays a role in adherence to human epithelial cells. Infect Immun. 2012;80(1):228-233. doi:10.1128/IAI.05913-11

7. Luo LM, Wu LJ, Xiao YL, et al. Enhancing pili assembly and biofilm formation in Acinetobacter baumannii ATCC19606 using non-native acyl-homoserine lactones. BMC Microbiol. 2015;15:62. doi:10.1186/ s12866-015-0397-5

8. Tomaras AP, Flagler MJ, Dorsey CW, Gaddy JA, Actis LA. Characterization of a two-component regulatory system from Acinetobacter baumannii that controls biofilm formation and cellular morphology. Microbiology (Reading, England). 2008;154(Pt 11):3398-3409. doi:10.1099/mic.0.2008/019471-0

9. Pakharukova N, Tuittila M, Paavilainen S, et al. Structural basis for Acinetobacter baumannii biofilm formation. Proc Natl Acad Sci USA. 2018;115(21):5558-5563. doi:10.1073/pnas.1800961115

10. Choi AH, Slamti L, Avci FY, Pier GB, Maira-Litrán T. The pgaABCD locus of Acinetobacter baumannii encodes the production of poly-beta-1-6-N-acetylglucosamine, which is critical for biofilm formation. $J$ Bacteriol. 2009;191(19):5953-5963. doi:10.1128/ JB.00647-09

11. Shenkutie AM, Yao MZ, Siu GK, Wong BKC, Leung PH. Biofilminduced antibiotic resistance in clinical Acinetobacter baumannii isolates. Antibiotics (Basel, Switzerland). 2020;9(11). doi:10.3390/ antibiotics9110817

12. Niu C, Clemmer KM, Bonomo RA, Rather PN. Isolation and characterization of an autoinducer synthase from Acinetobacter baumannii. J Bacteriol. 2008;190(9):3386-3392. doi:10.1128/JB.01929-07 
13. Nie D, Hu Y, Chen Z, et al. Outer membrane protein A (OmpA) as a potential therapeutic target for Acinetobacter baumannii infection. $J$ Biomed Sci. 2020;27(1):26. doi:10.1186/s12929-020-0617-7

14. Walsh BJC, Wang J, Edmonds KA, et al. The response of Acinetobacter baumannii to hydrogen sulfide reveals two independent persulfide-sensing systems and a connection to biofilm regulation. mBio. 2020;11(3). doi:10.1128/mBio.01254-20.

15. Clinical and Laboratory Standards Institute; Wayne PA. Performance Standards for Antimicrobial Susceptibility Testing, 30th Edition. CLSI M100. CLSI; 2020.

16. Ramirez MS, Bonomo RA, Tolmasky ME. Carbapenemases: transforming Acinetobacter baumannii into a yet more dangerous menace. Biomolecules. 2020;10(5):720. doi:10.3390/biom10050720

17. Krishnamoorthy S, Shah BP, Lee HH, Martinez LR. Microbicides alter the expression and function of RND-type efflux pump AdeABC in biofilm-associated cells of Acinetobacter baumannii clinical isolates. Antimicrob Agents Chemother. 2016;60(1):57-63. doi:10.1128/ AAC.01045-15

18. Stepanovic S, Vukovic D, Dakic I, Savic B, Svabic-Vlahovic M. A modified microtiter-plate test for quantification of staphylococcal biofilm formation. J Microbiol Methods. 2000;40(2):175-179. doi:10.1016/S0167-7012(00)00122-6

19. Pakkulnan R, Anutrakunchai C, Kanthawong S, Taweechaisupapong $\mathrm{S}$, Chareonsudjai P, Chareonsudjai S. Extracellular DNA facilitates bacterial adhesion during Burkholderia pseudomallei biofilm formation. PLoS One. 2019;14(3):e0213288. doi:10.1371/journal. pone. 0213288

20. Zhang B, Yu P, Wang Z, Alvarez PJJ. Hormetic promotion of biofilm growth by polyvalent bacteriophages at low concentrations. Environ Sci Technol. 2020;54(19):12358-12365. doi:10.1021/acs.est.0c03558

21. Tang M, Wei X, Wan X, Ding Z, Ding Y, Liu J. The role and relationship with efflux pump of biofilm formation in Klebsiella pneumoniae. Microb Pathog. 2020;147:104244. doi:10.1016/j. micpath.2020.104244

22. Moskowitz SM, Foster JM, Emerson J, Burns JL. Clinically feasible biofilm susceptibility assay for isolates of Pseudomonas aeruginosa from patients with cystic fibrosis. J Clin Microbiol. 2004;42(5):19151922. doi:10.1128/JCM.42.5.1915-1922.2004

23. Gao B, Li X, Yang F, et al. Molecular epidemiology and risk factors of ventilator-associated pneumonia infection caused by carbapenemresistant enterobacteriaceae. Front Pharmacol. 2019;10:262. doi:10.3389/fphar.2019.00262

24. Yang $\mathrm{CH}$, Su PW, Moi SH, Chuang LY. Biofilm formation in Acinetobacter baumannii: genotype-phenotype correlation. Molecules (Basel, Switzerland). 2019;24(10). doi:10.3390/molecules24101849

25. Bustin SA, Benes V, Garson JA, et al. The MIQE guidelines: minimum information for publication of quantitative real-time PCR experiments. Clin Chem. 2009;55(4):611-622. doi:10.1373/ clinchem.2008.112797

26. Livak KJ, Schmittgen TD. Analysis of relative gene expression data using real-time quantitative PCR and the 2(-Delta Delta C(T)) method. Methods (San Diego, Calif). 2001;25(4):402-408. doi:10.1006/meth.2001.1262

27. Wong D, Nielsen TB, Bonomo RA, Pantapalangkoor P, Luna B, Spellberg B. Clinical and pathophysiological overview of Acinetobacter infections: a century of challenges. Clin Microbiol Rev. 2017;30(1):409-447. doi:10.1128/CMR.00058-16

28. Wolcott R, Dowd S. The role of biofilms: are we hitting the right target? Plast Reconstr Surg. 2011;127(Suppl 1):28s-35s. doi:10.1097/PRS.0b013e3181fca244

29. Ni W, Han Y, Zhao J, et al. Tigecycline treatment experience against multidrug-resistant Acinetobacter baumannii infections: a systematic review and meta-analysis. Int J Antimicrob Agents. 2016;47(2):107116. doi:10.1016/j.ijantimicag.2015.11.011
30. Kengkla K, Kongpakwattana K, Saokaew S, Apisarnthanarak A, Chaiyakunapruk N. Comparative efficacy and safety of treatment options for MDR and XDR Acinetobacter baumannii infections: a systematic review and network meta-analysis. $J$ Antimicrob Chemother. 2018;73(1):22-32. doi:10.1093/jac/dkx368

31. Cai Y, Lee W, Kwa AL. Polymyxin B versus colistin: an update. Expert Rev Anti Infect Ther. 2015;13(12):1481-1497. doi:10.1586/ 14787210.2015.1093933

32. Garnacho-Montero J, Timsit JF. Managing Acinetobacter baumannii infections. Curr Opin Infect Dis. 2019;32(1):69-76. doi:10.1097/ QCO.0000000000000518

33. Qi L, Li H, Zhang C, et al. Relationship between antibiotic resistance, biofilm formation, and biofilm-specific resistance in Acinetobacter baumannii. Front Microbiol. 2016;7:483. doi:10.3389/fmicb.2016.00483

34. Gaddy JA, Actis LA. Regulation of Acinetobacter baumannii biofilm formation. Future Microbiol. 2009;4(3):273-278. doi:10.2217/ fmb.09.5

35. Khoshnood S, Savari M, Abbasi Montazeri E, Farajzadeh Sheikh A. Survey on genetic diversity, biofilm formation, and detection of colistin resistance genes in clinical isolates of Acinetobacter baumannii. Infect Drug Resist. 2020;13:1547-1558. doi:10.2147/IDR. S253440

36. Lin MF, Lin YY, Lan CY. Characterization of biofilm production in different strains of Acinetobacter baumannii and the effects of chemical compounds on biofilm formation. PeerJ. 2020;8:e9020. doi: 10.7717 peerj.9020

37. Rodríguez-Baño J, Martí S, Soto S, et al. Biofilm formation in Acinetobacter baumannii: associated features and clinical implications. Clin Microbiol Infect. 2008;14(3):276-278. doi:10.1111/ j.1469-0691.2007.01916.x

38. Shi N, Gao Y, Yin D, et al. The effect of the sub-minimal inhibitory concentration and the concentrations within resistant mutation window of ciprofloxacin on MIC, swimming motility and biofilm formation of Pseudomonas aeruginosa. Microb Pathog. 2019;137:103765. doi:10.1016/j.micpath.2019.103765

39. Stewart PS. Theoretical aspects of antibiotic diffusion into microbial biofilms. Antimicrob Agents Chemother. 1996;40(11):2517-2522. doi:10.1128/AAC.40.11.2517

40. Ciofu O, Rojo-Molinero E, Macià MD, Oliver A. Antibiotic treatment of biofilm infections. APMIS. 2017;125(4):304-319. doi:10.1111/apm.12673

41. Jensen P, Briales A, Brochmann RP, et al. Formation of hydroxyl radicals contributes to the bactericidal activity of ciprofloxacin against Pseudomonas aeruginosa biofilms. Pathog Dis. 2014;70 (3):440-443. doi:10.1111/2049-632X.12120

42. De Groote VN, Verstraeten N, Fauvart M, et al. Novel persistence genes in Pseudomonas aeruginosa identified by high-throughput screening. FEMS Microbiol Lett. 2009;297(1):73-79. doi:10.1111/ j.1574-6968.2009.01657.x

43. McQueary CN, Actis LA. Acinetobacter baumannii biofilms: variations among strains and correlations with other cell properties. $J$ Microbiol (Seoul, Korea). 2011;49(2):243-250. doi:10.1007/s12275011-0343-7

44. Tomaras AP, Dorsey CW, Edelmann RE, Actis LA. Attachment to and biofilm formation on abiotic surfaces by Acinetobacter baumannii: involvement of a novel chaperone-usher pili assembly system. Microbiology (Reading, England). 2003;149(12):3473-3484. doi:10.1099/mic.0.26541-0

45. Selasi GN, Nicholas A, Jeon H, et al. Differences in biofilm mass, expression of biofilm-associated genes, and resistance to desiccation between epidemic and sporadic clones of carbapenem-resistant Acinetobacter baumannii sequence type 191. PLoS One. 2016;11 (9):e0162576. doi:10.1371/journal.pone.0162576 
46. Smani Y, Fàbrega A, Roca I, Sánchez-Encinales V, Vila J, Pachón J. Role of OmpA in the multidrug resistance phenotype of Acinetobacter baumannii. Antimicrob Agents Chemother. 2014;58 (3):1806-1808. doi:10.1128/AAC.02101-13

47. Sánchez-Encinales V, Álvarez-marín R, Pachón-Ibáñez ME, et al. Overproduction of outer membrane protein A by Acinetobacter baumannii as a risk factor for nosocomial pneumonia, bacteremia, and mortality rate increase. $J$ Infect Dis. 2017;215(6):966-974. doi:10.1093/infdis/jix010

48. Martín-Peña R, Domínguez-Herrera J, Pachón J, McConnell MJ. Rapid detection of antibiotic resistance in Acinetobacter baumannii using quantitative real-time PCR. J Antimicrob Chemother. 2013;68 (7):1572-1575. doi:10.1093/jac/dkt057
49. Mayer C, Muras A, Romero M, López M, Tomás M, Otero A. Multiple quorum quenching enzymes are active in the nosocomial pathogen Acinetobacter baumannii ATCC17978. Front Cell Infect Microbiol. 2018;8:310. doi:10.3389/fcimb.2018.00310

50. Altınok Ö, Boral B, Ergin A, Eser ÖK. Existence of biofilm and biofilm-associated virulence genes in multi-drug resistant invasive Acinetobacter baumannii isolates. Mikrobiyol Bul. 2020;54(1):4049. doi:10.5578/mb.20204

51. Liu H, Wu YQ, Chen LP, et al. Biofilm-related genes: analyses in multi-antibiotic resistant Acinetobacter baumannii isolates from Mainland China. Medical Sci Monit. 2016;22:1801-1807. doi:10.12659/MSM.898959

\section{Publish your work in this journal}

Infection and Drug Resistance is an international, peer-reviewed openaccess journal that focuses on the optimal treatment of infection (bacterial, fungal and viral) and the development and institution of preventive strategies to minimize the development and spread of resistance. The journal is specifically concerned with the epidemiology of antibiotic resistance and the mechanisms of resistance development and diffusion in both hospitals and the community. The manuscript management system is completely online and includes a very quick and fair peerreview system, which is all easy to use. Visit http://www.dovepress.com/ testimonials.php to read real quotes from published authors. 